\title{
Characterizing acetogenic metabolism using a genome-scale metabolic reconstruction of Clostridium ljungdahlii
}

\author{
Harish Nagarajan ${ }^{1}$, Merve Sahin ${ }^{1}$, Juan Nogales ${ }^{1}$, Haythem Latif ${ }^{1}$, Derek R Lovley², Ali Ebrahim \\ and Karsten Zengler ${ }^{1 *}$
}

\begin{abstract}
Background: The metabolic capabilities of acetogens to ferment a wide range of sugars, to grow autotrophically on $\mathrm{H}_{2} / \mathrm{CO}_{2}$, and more importantly on synthesis gas $\left(\mathrm{H}_{2} / \mathrm{CO} / \mathrm{CO}_{2}\right)$ make them very attractive candidates as production hosts for biofuels and biocommodities. Acetogenic metabolism is considered one of the earliest modes of bacterial metabolism. A thorough understanding of various factors governing the metabolism, in particular energy conservation mechanisms, is critical for metabolic engineering of acetogens for targeted production of desired chemicals.

Results: Here, we present the genome-scale metabolic network of Clostridium ljungdahlii, the first such model for an acetogen. This genome-scale model (iHN637) consisting of 637 genes, 785 reactions, and 698 metabolites captures all the major central metabolic and biosynthetic pathways, in particular pathways involved in carbon fixation and energy conservation. A combination of metabolic modeling, with physiological and transcriptomic data provided insights into autotrophic metabolism as well as aided the characterization of a nitrate reduction pathway in $C$. ljungdahlii. Analysis of the iHN637 metabolic model revealed that flavin based electron bifurcation played a key role in energy conservation during autotrophic growth and helped identify genes for some of the critical steps in this mechanism.

Conclusions: iHN637 represents a predictive model that recapitulates experimental data, and provides valuable insights into the metabolic response of $C$. ljungdahlii to genetic perturbations under various growth conditions. Thus, the model will be instrumental in guiding metabolic engineering of C. ljungdahlii for the industrial production of biocommodities and biofuels.
\end{abstract}

\section{Background}

Acetogenic microorganisms have unique metabolic capabilities that, if understood, could be harnessed to greatly increase strain engineering design options for microbial production of biofuels and biocommodities. Acetogens were discovered for their ability to autotrophically reduce $\mathrm{CO}_{2}$ to acetate and conserve energy simultaneously using the Wood-Ljungdahl pathway [1]. In addition to reducing $\mathrm{CO}_{2}$, acetogens can ferment a wide variety of sugars as well. Their ability for autptrophy allows them also to grow on synthesis gas $\left(\mathrm{H}_{2} / \mathrm{CO} / \mathrm{CO}_{2}\right)$ by utilizing either $\mathrm{H}_{2} / \mathrm{CO}_{2}$

\footnotetext{
* Correspondence: kzengler@ucsd.edu

${ }^{1}$ Department of Bioengineering, University of California San Diego, La Jolla, CA, USA

Full list of author information is available at the end of the article
}

or CO alone. More recently, acetogens like Sporomusa ovata and Clostridium ljungdahlii have been shown to be capable of another form of autotrophic metabolism, called microbial electrosynthesis [2]. Microbial electrosynthesis is a process in which microorganisms directly use electric current to reduce carbon dioxide to multi-carbon organic compounds that are excreted from the cells into extracellular medium [3]. These discoveries further expand the range of economically viable feedstocks that can be used for industrial production of biofuels and biochemicals [4].

However, to efficiently engineer acetogens into platform strains and production hosts for chemicals at an industrial scale, a thorough understanding of the metabolic capabilities and aspects of energy conservation is a necessary prerequisite. The Wood-Ljungdahl pathway of
C Biomed Central

(C) 2013 Nagarajan et al.; licensee BioMed Central Ltd. This is an open access article distributed under the terms of the Creative Commons Attribution License (http://creativecommons.org/licenses/by/2.0), which permits unrestricted use, distribution, and reproduction in any medium, provided the original work is properly cited. 
carbon fixation employed by acetogens is believed to be one of the most ancient metabolic pathways [5]. Physiological and biochemical aspects governing this metabolic capability have been poorly characterized with several fundamental discoveries being made only recently [6,7]. One of the fundamental characteristics of acetogenic metabolism discovered recently is the concept of flavin-based electron bifurcation. In this mechanism, there is a concomitant coupling of an endergonic redox reaction with the oxidation of the same electron donor with higher potential electron acceptors. This feature is believed to play a key role in the energy conservation mechanisms of acetogens [8].

Constraints based reconstruction and analysis (COBRA) has been a powerful technique for discovering and understanding new capabilities and content in microorganisms, as well as in guiding metabolic engineering efforts for targeted production [9]. The COBRA approach relies on a genome-scale metabolic network reconstruction, which enumerates the metabolic transformations and the genes encoding them in a mathematical format. This reconstructed network together with physiological data can then enable the prediction of the functionality of an organism under conditions of interest. Such a validated and accurate network can be utilized for prospective design and engineering of cellular networks [10]. Furthermore, genome-scale metabolic networks of bacteria and constraints-based modeling have been instrumental in guiding metabolic engineering at an industrial scale [11].

In this study, we reconstructed the first genome-scale metabolic network of an acetogen, Clostridium ljungdahlii. We characterized the metabolic phenotypes of this bacterium under heterotrophic and autotrophic growth conditions. We further utilized physiological and transcriptomic data to elucidate novel biological capabilities and aspects of energy conservation during autotrophic metabolism of C. ljungdahlii.

\section{Results and discussion}

Genome-scale reconstruction of the acetogen Clostridium ljungdahlii

The genome-scale metabolic network for C. ljungdahlii was reconstructed using a four-step integrative reconciliatory workflow involving four published models of related clostridia species and two draft models (Figure 1). The first draft metabolic model was generated based on the $C$. ljungdahlii genome annotation [12] using the AutoModel functionality of SimPheny (Genomatica, San Diego), while the second draft model was generated using the ModelSEED database [13]. In addition to these two draft models, homologs to $C$. ljungdahlii genes were identified in published genome-scale reconstructions of related clostridia species (C. acetobutylicum, C. thermocellum, and
C. beijerenckii) [14-17] using the Smith-Waterman alignment. A $60 \%$ amino acid sequence identity cutoff was used to identify $C$. ljungdahlii homologs in the other clostridia genomes. The reactions corresponding to these genes in the respective clostridia models were compiled and reconciled with the two draft models. The list of discrepancies pertaining to nomenclature between the different databases and gene-reaction associations was manually curated with the aid of biochemical literature and databases such as KEGG [18] and SEED [13]. Manual evaluation of new content from the annotation and existing genome-scale reconstructions consisted of gathering genetic, biochemical, sequence, and physiological data and reconciling this information to determine the likelihood of each reaction being present in the organism. The curated reconstruction was evaluated for functional performance with the aid of a biomass objective function that was formulated using an existing template (see Methods). Using inference based on pathway function, as well as the SMILEY computational algorithm $[19,20]$, which predicts reactions that fill gaps in a metabolic network, reaction content was added to the network so that it could produce the necessary biomass components. This resulted in a final network (iHN637) consisting 637 genes, 785 reactions, and 698 metabolites. This iHN637 reconstruction represents the first genome-scale metabolic model of an acetogen.

\section{Functional testing of the model}

The functional capabilities of the iHN637 reconstruction were evaluated by simulating growth under different conditions using the developed biomass objective function (Methods). iHN637 was successfully able to simulate heterotrophic growth on several of the known substrates [21] (fructose, glucose, gluconate, arabinose, ribose, xylose, ethanol, formate, serine, citrulline, pyruvate, arginine, aspartate, and glutamate). Moreover, autotrophic growth with $\mathrm{H}_{2}$ and $\mathrm{CO}_{2}$ as well as just $\mathrm{CO}$ alone was feasible using the iHN637 reconstruction. The predicted growth rates and acetate production rates for each of these various substrates are provided in Table 1. In addition to acetate and ethanol, the iHN637 metabolic model is also capable of producing lactate and 2,3-butanediol using native pathways as described elsewhere [22]. Furthermore, the model was able to successfully predict a growth rate consistent with experimental observations for heterotrophic growth on fructose. When the iHN637 model was constrained with experimentally determined uptake rates of fructose (1.8 $\mathrm{mmol} / \mathrm{gDWh})$, the growth rate predicted by the model $\left(0.077 \mathrm{~h}^{-1}\right)$ was in good agreement with the experimentally measured growth rates $\left(0.072 \mathrm{~h}^{-1}\right)$. The simulated acetate production rate $(2.7 \mathrm{mmol} / \mathrm{gDWh})$ was also 

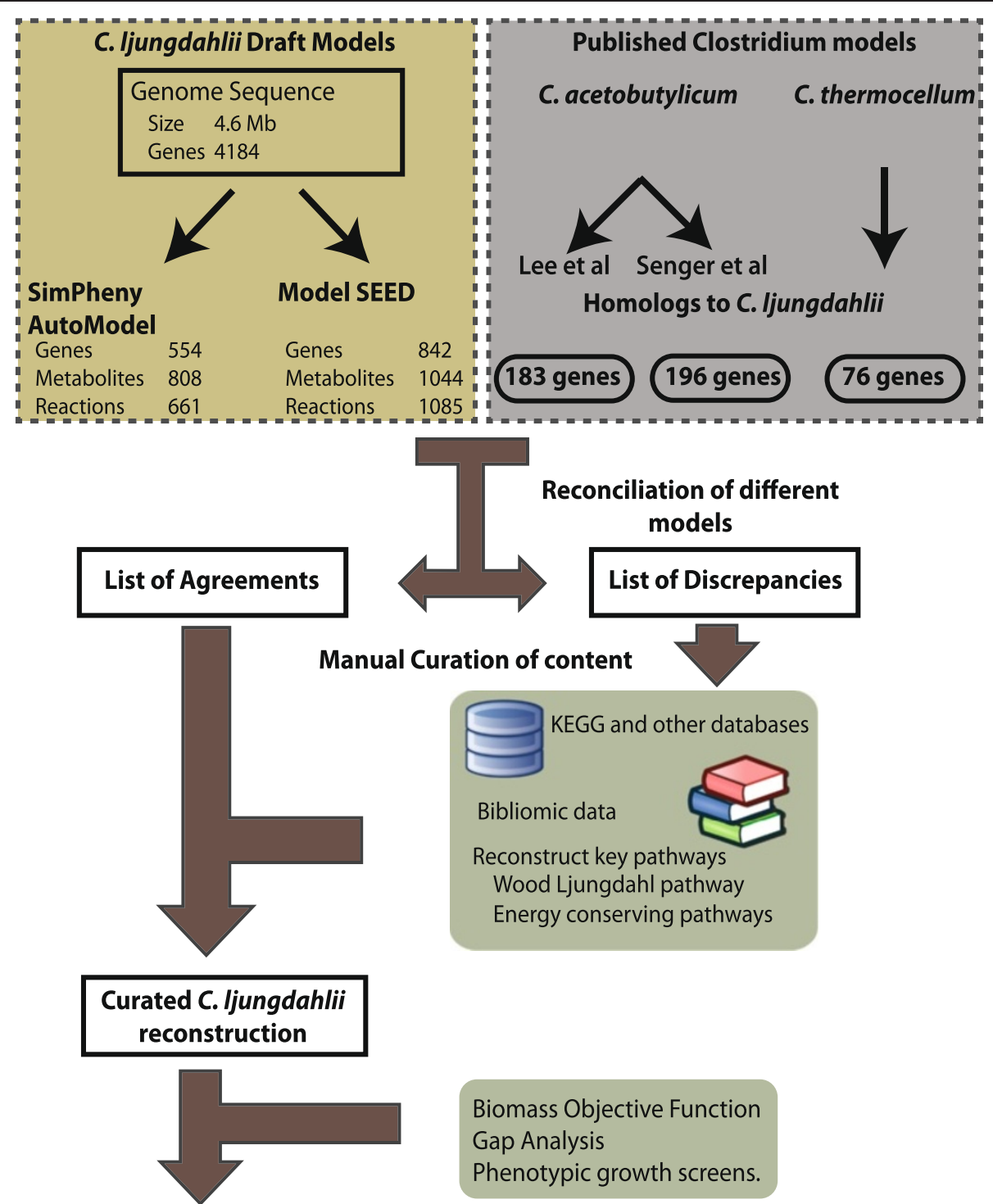

\begin{tabular}{|lc|}
\hline $\begin{array}{l}\text { Functional metabolic model of } \\
\text { C. ljungdahlii }\end{array}$ \\
\hline Genes & 637 \\
Metabolites & 698 \\
Reactions & 785 \\
\hline
\end{tabular}

Figure 1 Reconstruction workflow. Iterative reconciliatory reconstruction workflow adopted for generating the metabolic model for C. ljungdahlii.

consistent with the measured value of $2.85 \mathrm{mmol} / \mathrm{gDWh}$ (Additional file 1: Figure S1).

Characterization of a nitrate reduction pathway in Clostridium ljungdahlii

A novel nitrate reduction pathway for clostridia was reconstructed in the iHN637 model based on the C. ljungdahlii genome annotation. In addition to the glutamine syn- thetase and glutamine:2-oxoglutarate aminotransferase or the fixation of dinitrogen using a molybdenum-dependent nitrogenase, this pathway could serve as a third possible nitrogen assimilation route in C. ljungdahlii. This pathway presents characteristics of both, assimilatory and respiratory nitrate reductases and the reduction of nitrate to ammonia via hydroxylamine resembles the nitrate respiratory system recently identified in Nautilia profundicola [23]. 
Table 1 Model predicted growth rates and acetate production rates using iHN637

\begin{tabular}{cccc}
\hline Substrate & $\begin{array}{c}\text { Substrate } \\
\text { uptake rate } \\
(\mathbf{m m o l} / \mathbf{g D W} / \mathbf{h})\end{array}$ & $\begin{array}{c}\text { Specific growth } \\
\text { rate } \mathbf{( 1 / h )}\end{array}$ & $\begin{array}{c}\text { Acetate } \\
\text { production rate } \\
\text { (mmol/gDW/h) }\end{array}$ \\
\hline Fructose & 1.88 & 0.077 & 2.70 \\
Fructose & 5 & 0.224 & 9.30 \\
Glucose & 5 & 0.212 & 9.61 \\
Gluconate & 5 & 0.191 & 8.91 \\
Arabinose & 5 & 0.174 & 8.09 \\
Ribose & 5 & 0.133 & 9.12 \\
Xylose & 5 & 0.174 & 8.09 \\
Formate & 5 & 0.011 & 0.96 \\
Serine & 5 & 0.074 & 4.36 \\
Citrulline & 5 & 0.034 & 4.14 \\
Pyruvate & 5 & 0.063 & 4.65 \\
Arginine & 5 & 0.035 & 4.13 \\
Asparatate & 5 & 0.097 & 5.05 \\
Glutamate & 5 & 0.108 & 8.51 \\
CO ${ }_{\mathbf{2}}\left(\mathrm{H}_{\mathbf{2}}\right)$ & $10(20)$ & 0.034 & 4.14 \\
CO & 20 & 0.06 & 3.48 \\
\hline
\end{tabular}

The substrate uptake rates listed in the table was used to constrain the model.

The nitrate reduction pathway consists of a soluble nitrate reductase (NTRARf), a nitrite reductase (NTRIR5), and an additional hydroxylamine reductase (HAMR) (Figure 2A). Transcriptomic profiling of C. ljungdahlii grown heterotrophically on fructose in medium containing ammonium or ammonium-free medium with nitrate as a nitrogen source, helped validate this pathway. The genes encoding for the nitrate reduction pathway were all significantly upregulated (>8-fold) when nitrate was provided in ammonium-free medium. Specifically, the genes encoding for nitrate reductase, nitrite reductase, and the nitrate transporter were upregulated greater than 400-fold (Figure 2A). Growth experiments showed that nitrate was consumed with stoichiometric production of ammonium (Figure 2B), further validating the proposed pathway. Furthermore, modeling simulations predict a $15 \%$ reduction in acetate flux when nitrate served as nitrogen source due to nitrate reduction acting as an additional electron sink (Additional file 2: Table S1). The diversion of electrons seems to impact the acetate production gained through fixation of $\mathrm{CO}_{2}$ using electrons obtained from fermenting fructose. In addition, nitrate reduction has been thought of as a primitive mode of energy metabolism in certain clostridia with potential roles in balancing electrons and affecting growth yields during fermentation [24]. The identification of this pathway provides the opportunity for a detailed investigation on its potential functional effects on the fermentation of C. ljungdahlii.

\section{Reconstructing the carbon fixation pathway in C. ljungdahlii}

The C. ljungdahlii genome encodes for all the genes involved in the Wood-Ljungdahl pathway of carbon fixation [12]. Accordingly, this pathway was incorporated into the iHN637 reconstruction. The pathway consists of two different branches, one $\mathrm{CO}_{2}$ contributing to the methyl group of acetyl CoA via the eastern branch and the other $\mathrm{CO}_{2}$ contributing to the carbonyl group of the acetyl CoA via the western branch [25]. The key enzyme in this pathway is the CODH/ACS complex, which performs the dual activity of reducing $\mathrm{CO}_{2}$ to $\mathrm{CO}$ via the carbon monoxide dehydrogenase $(\mathrm{CODH})$ activity and the subsequent formation of acetyl CoA through the acetyl-CoA synthase (ACS). It has been suggested that the $\mathrm{CODH} / \mathrm{ACS}$ complex functions in a manner that $\mathrm{CO}$ resulting from $\mathrm{CODH}$ activity is kept as a bound metabolite in the complex to diminish thermodynamic barriers involved in this energy intensive process [26]. Furthermore, the genome of $C$. ljungdahlii suggested that $\mathrm{CO}$ oxidation to $\mathrm{CO}_{2}$ is likely to be catalyzed by a different carbon monoxide dehydrogenase gene (CLJU c0909009110) other than the ones encoded by the CODH/ACS complex. Taking these into consideration, the iHN637 reconstruction incorporates the $\mathrm{CODH} / \mathrm{ACS}$ reaction as the net reaction of carbon monoxide dehydrogenase and acetyl CoA synthase activity, and associates the gene cluster CLJU c09090-09110 to the reaction representing CO oxidation to $\mathrm{CO}_{2}$. The methylenetetrahydrofolate dehydrogenase reaction catalyzed by the bifunctional FolCD (CLJU_c37630) was assumed to be NADPH-dependent based on sequence similarity with the corresponding enzyme in the acetogen Moorella thermoacetica [25,27]. The energy conservation mechanisms associated with autotrophic growth using the Wood-Ljungdahl pathway is discussed in detail in the following section.

\section{Analysis of energy conservation steps in C. ljungdahlii}

Acetogens have long been thought to be living at the thermodynamic limit due to the energy requirements of the Wood-Ljungdahl pathway [7]. Given that the ATP generated from acetate production is required for the activation of formate, it has been proposed that acetogens should have additional energy conservation mechanisms [7]. Acetogens have generally been classified into those containing respiratory cytochromes that establish a proton gradient (e.g., M. thermoacetica) and those that do not. Organisms such as Acetobacterium woodii have been shown to employ a sodium gradient as an energy conservation mechanism [7]. The C. ljungdahlii genome sequence has revealed that it falls into a third class of acetogens that neither uses respiratory cytochromes nor a sodium gradient for energy conservation [12]. As reviewed recently, flavin-based electron bifurcation is 


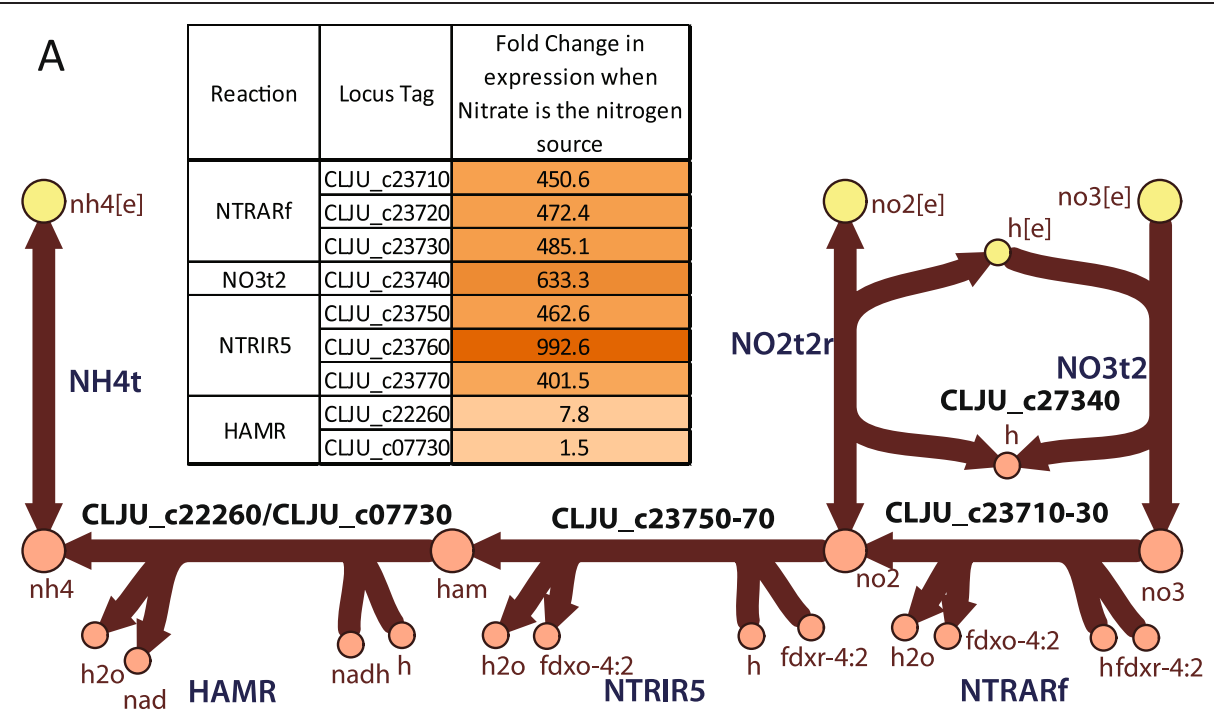

B

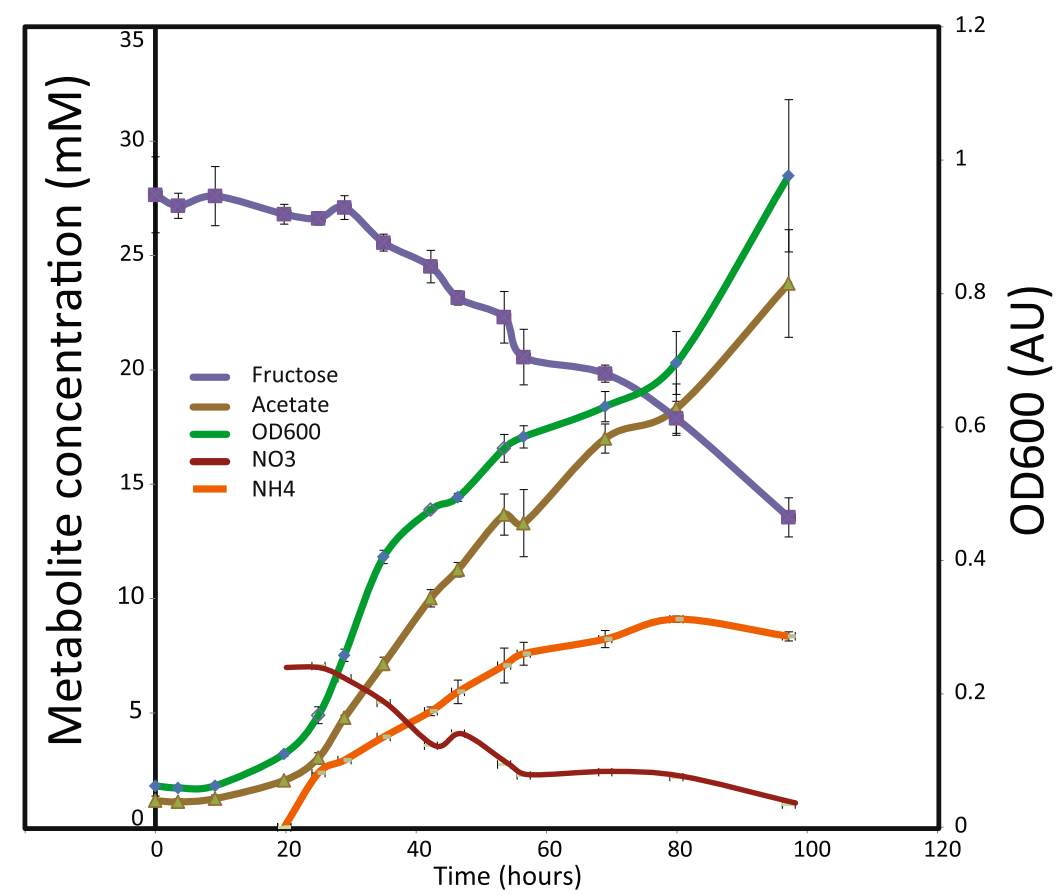

Figure $\mathbf{2}$ Characterizing the nitrate reduction pathway in C. ljungdahlii. (A) Pathway map of the reconstructed nitrate reduction pathway with the respective genes associated to the reactions. Inset shows the differential expression of the genes in the nitrate reduction pathway under ammonium-free medium with nitrate compared to nitrate-free medium with ammonium as the nitrogen source. Reaction abbreviations: NO3t2 (nitrate transporter), NO2t2r (nitrite transporter), NTRARf (nitrate reductase), NTRIR5 (nitrite reductase), HAMR (hydroxylamine reductase), NH4t (ammonium transporter). (B) Physiological data when C. ljungdahlii is grown on fructose in ammonium-free media with nitrate as the nitrogen source. Purple line represents fructose, green line represents $\mathrm{OD}_{600}$, brown line represents acetate, red line represents nitrate and orange line represents ammonia measurements respectively.

expected to serve as an alternative mechanism of energy conservation in acetogens [8]. Analysis of the iHN637 reconstruction of C. ljungdahlii reveals that electron bifurcation and proton translocating ferredoxin oxidation are critical mechanisms for energy conservation during autotrophic growth in C. ljungdahlii (Figure 3). The iHN637 model predicted proton translocation by the membranebound Rnf complex while oxidizing ferredoxin and reducing NAD was essential for autotrophic growth. Specifically, the proton gradient established by the Rnf complex is essential for driving ATP generation through ATP synthase (Figure 3B). This prediction is consistent with recent 

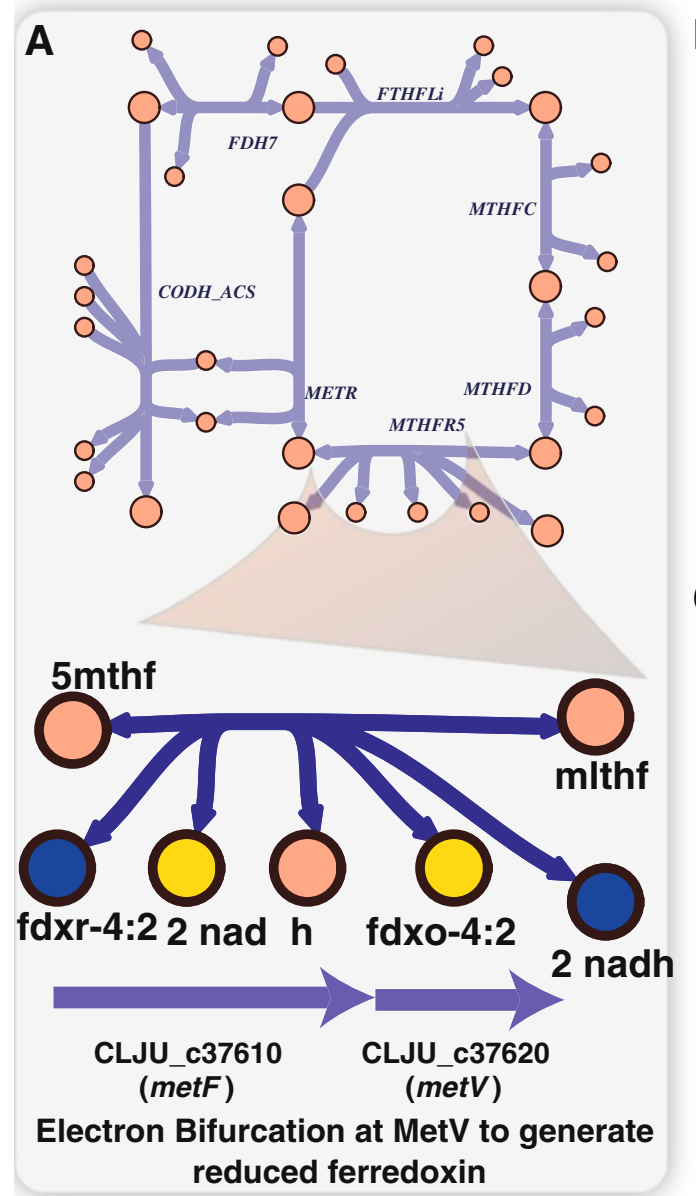

D

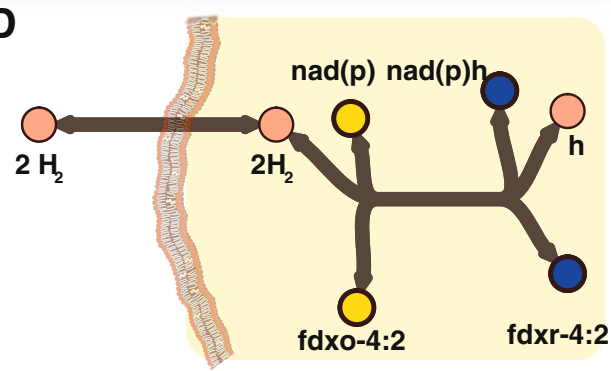

B

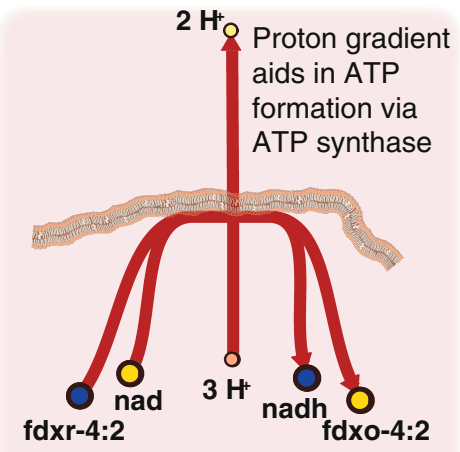

Energy Conservation via Rnf complex (CLJU_c11360-11410)

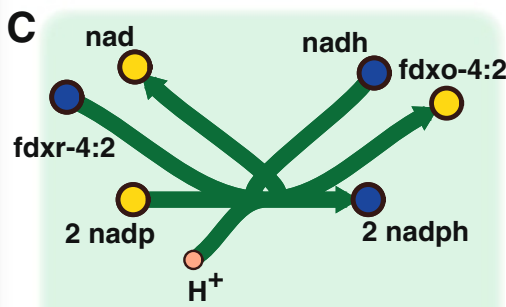

NADPH generated through the electron bifurcating Nfn complex CLJU_c37240

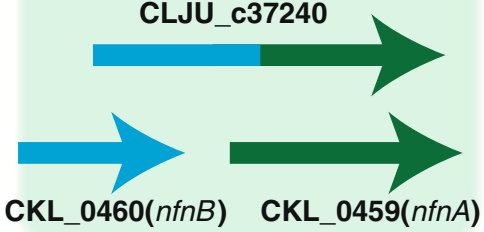

Electron bifurcating hydrogenase for reduction of ferredoxin from hydrogen

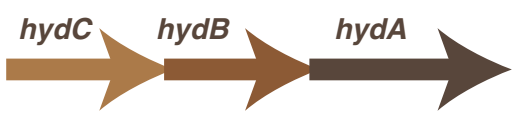

CLJU_c07030 CLJU_c07040 CLJU_c07070

Figure 3 Energy conservation mechanisms in C. ljungdahlii. (A) Shown is the Wood-Ljungdahl pathway with an emphasis on the electron bifurcation in the Wood-Ljungdahl pathway at the methylene-tetrahydrofolate deydrogenase small subunit to generate reduced ferredoxin. The detailed Wood-Ljungdahl pathway is shown in Figure 4. Reaction abbreviations are the same as those in Figure 4. (B) Energy conservation through proton gradient established at the Rnf complex. (C) Identification of a gene encoding the electron bifurcating transhydrogenase for the generation of NADPH during autotrophic growth (Nfn complex). (D) Electron bifurcating hydrogenase for the reduction of ferredoxin from hydrogen during autotrophic growth. Yellow nodes indicate the oxidized form of reducing equivalents and blue nodes indicate the reduced form.

experimental observations of the lack of autotrophic growth in a strain where the Rnf complex was deleted [28].

An electron-bifurcating transhydrogenase (Nfn complex) that couples exergonic reduction of NADP with ferredoxin to drive the endergonic reduction of NADP with NADH had been characterized in C. kluyveri [29]. Recently, a similar electron bifurcating NADPH transhydrogenase activity was also reported in the acetogen $M$. thermoacetica [30]. A homolog to the $n f n A$ and $n f n B$ genes in C. kluyveri was identified in the C. ljungdahlii genome. This gene (CLJU_c37240) was previously annotated as glutamate synthase. Another recent report describes a similar electron-bifurcating NADPH transhydrogenase activity in $C$. autoethanogenum and further indicates the presence of a homolog in C. ljungdahlii [31]. As part of iHN637, this gene (CLJU_c37240) has 
been assigned the electron bifurcating transhydrogenase reaction (Figure $3 \mathrm{C}$ ). Simulatons further reveal that this reaction is essential for the interconversion between $\mathrm{NADPH}$ and NADH during autotrophic growth.

It was speculated that the highly exergonic reduction of methylene-tetrahydrofolate by NADH could be a site for electron bifurcation to generate additional reduced ferredoxin from NADH [12]. Investigation of the $A$. woodii genome revealed that the small subunit of the methylenetetrahydrofolate reductase (MetV) can act as a flavoprotein that could aid in this proposed electron bifurcation, thereby indirectly establishing a proton gradient through the Rnf complex [7]. A homolog to the MetV gene from A. woodii was identified next to the MetF gene in C. ljungdahlii and investigation of the intergenic spacing in the genome revealed a possible organization of MetV and MetF in the same operon (CLJU c37610 and CLJU c37620). Hence, an electron bifurcating methylenetetrahydrofolate reductase reaction was included in the iHN637 reconstruction (Figure 3A).

\section{Modeling of heterotrophic and autotrophic metabolism} Transcriptome profiling of $C$. ljungdahlii when grown on fructose and autotrophically on $\mathrm{H}_{2} / \mathrm{CO}_{2}$ identified 114 differentially expressed genes (50 upregulated and 64 downregulated in autotrophic conditions) (Additional file 3: Table S2). Among the key genes that were downregulated during autotrophic growth were genes involved in fructose metabolism, pyrimidine biosynthesis, and amino acid biosynthesis. The downregulation of biosynthetic genes in autotrophic conditions is likely due to the slower growth rate during autotrophic growth compared to heterotrophic growth. Among the key genes upregulated was the cooS1 (CODH) gene. The cooS1 gene in Carboxydothermus hydrogenoformans is hypothesized to play a role in facilitating electron transfer through the hydrogenase [32]. Hence it is possible that this gene in C. ljungdahlii has a similar role while growing on hydrogen. Importantly, transcriptome profiling during autotrophic growth revealed that the gene annotated as met $V$ was co-transcribed along with $m e t F$, further validating the annotation and assignment of its function for electron bifurcation with metF (Additional file 4: Figure S2). It must also be noted that genes encoding the Wood-Ljungdahl pathway are not differentially expressed between the conditions examined in this study (heterotrophic growth on fructose versus autotrophic growth on $\mathrm{H}_{2} / \mathrm{CO}_{2}$ ). This is probably due to the fact that the Wood-Ljungdahl pathway is employed for fixation of $\mathrm{CO}_{2}$ using electrons obtained from fermenting sugars [1]. However, a recent transcriptomic study on syngas utilization in C. ljungdahlii showed upregulation of the genes encoding the Wood-Ljungdahl pathway under autotrophic growth (4:1 $\left.\mathrm{CO}: \mathrm{CO}_{2}\right)$ [33]. This difference can be attributed to the differences in the autotrophic conditions investigated in both these studies with one being $\mathrm{CO}_{2} / \mathrm{H}_{2}$ and the other being a condition rich in $\mathrm{CO}$.

The genome-scale model of $C$. ljungdahlii has revealed that flavin-based electron bifurcation is found to play a critical role in the essential reactions for autotrophic growth, thereby providing an explicit account of the requirement of reducing equivalents for the fixation of $\mathrm{CO}_{2}$ to acetyl CoA (Figure 4A). Another instance of electron bifurcation in the autotrophic growth of C. ljungdahlii was observed in the first step of $\mathrm{H}_{2}$ activation to reduce ferredoxin. A bifurcating hydrogenase (HydABC), which couples the exergonic reduction of $\mathrm{NADH}$ from $\mathrm{H}_{2}$ to drive the endergonic reduction of ferredoxin from $\mathrm{H}_{2}$ has been identified to perform this process in $A$. woodii $[7,34]$. A homolog to this gene cluster has been identified in $C$. ljungdahlii (Figure 3D). However, in another recent report, a similar electron-bifurcating hydrogenase that is specific to NADPH has been identified and characterized in $C$. autoethanogenum, an acetogen closely related to $C$. ljungdahlii [31]. This report also mentions that the hydrogenase of $C$. ljungdahlii is homologous to the NADPspecific hydrogenase in C. autoethanogenum. Since there is no definitive biochemical data available for C. ljungdahlii the metabolic reconstruction (iHN637) has both the NAD-specific and NADP-specific electron bifurcating hydrogenases associated to this gene cluster, with the NADP-specific one being set as the default.

The impact of this cofactor specificity on the metabolic network and the importance of detailing these energy conservation mechanisms was further highlighted when genetic perturbations were modeled under heterotrophic and autotrophic conditions. Specifically, the effect of knocking out acetate kinase $(a c k A)$ was modeled under heterotrophic growth on fructose and autotrophic growth under the major constituents of syngas ( $\mathrm{CO}$ alone as well as $\mathrm{H}_{2} / \mathrm{CO}_{2}$ ) (Figure 5). The deletion of $a c k A$ is one of the primary targets for optimizing ethanol production in acetogens.

Modeling simulations revealed that the activity of acetate kinase was not essential for growth on fructose. As expected, the lost ATP from the acetate kinase reaction adversely impacted the growth resulting in a $20 \%$ reduction in growth yield with the model predicting a diversion of flux to ethanol (Figure 5A).

When ethanol is the desired product from carbon fixation through the Wood-Ljungdahl pathway, 2 reducing equivalents in the form of $\mathrm{NADH}$ are required in addition to the 4 reducing equivalents ( $1 \mathrm{NADPH}, 1$ ferredoxin, and $2 \mathrm{NADH}$ ) and 1 ATP (Figure 4B). Since ethanol production from syngas is an industrially relevant process, iHN637 was used to simulate the effect of this important deletion (ackA) under two different autotrophic growth conditions ( $\mathrm{CO}$ alone as well as $\left.\mathrm{H}_{2} / \mathrm{CO}_{2}\right)$ that reflect the composition of syngas. 


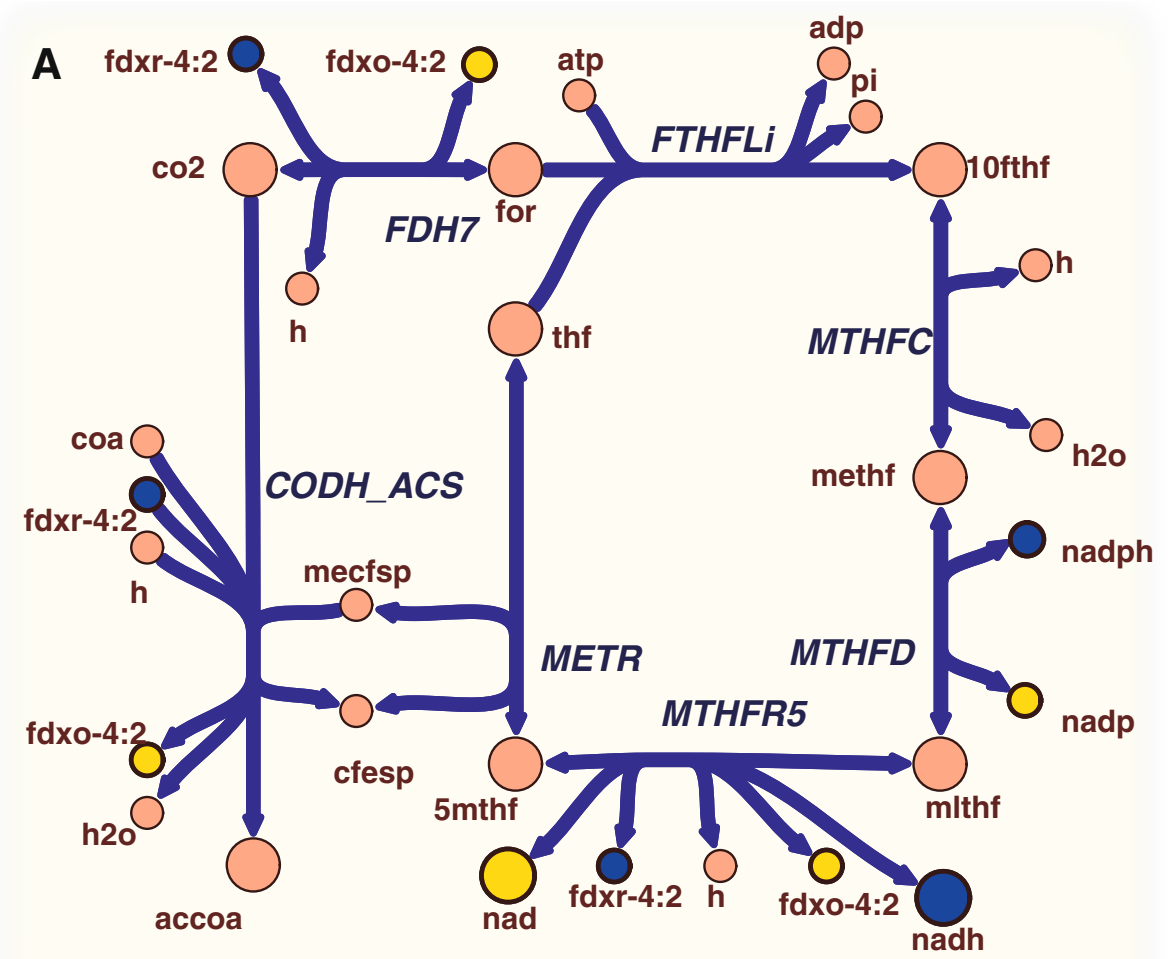

1 ATP and 4 reducing equivalents ( $1 \mathrm{fdxr}-4: 2,1$ nadph, and 2 nadh) required to fix $2 \mathrm{CO}_{2}$ to 1 accoa

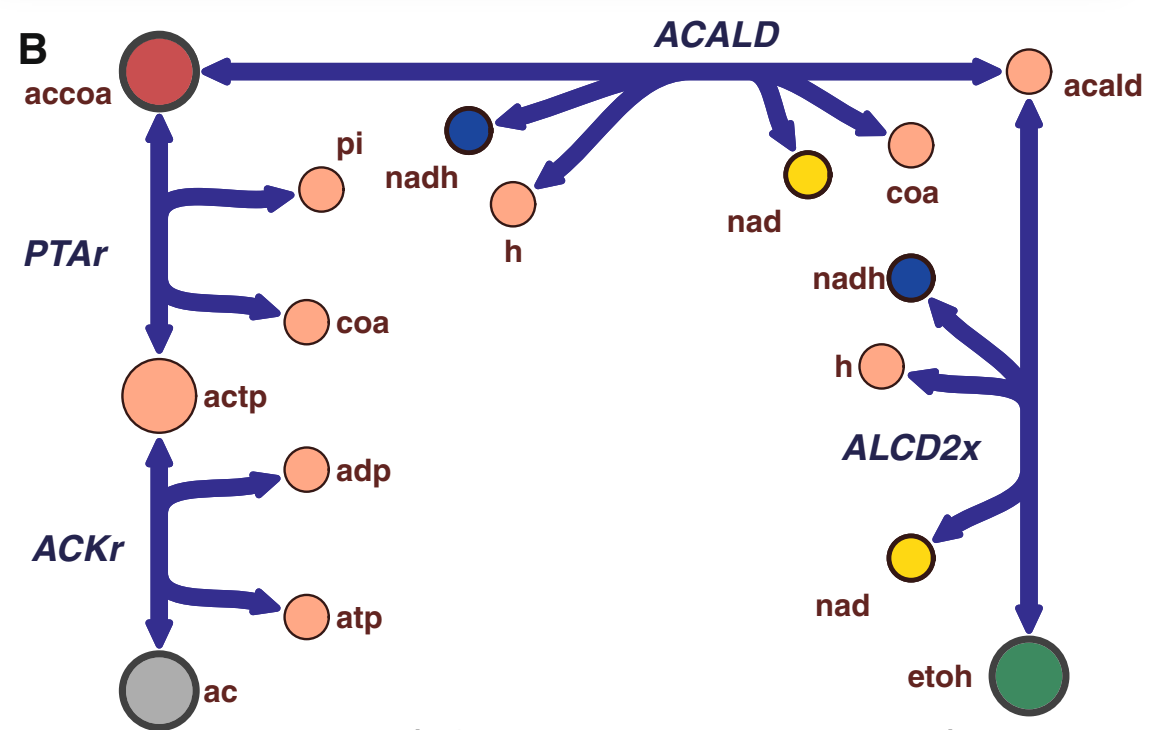

4 reducing equivalents ( $1 \mathrm{fdxr}-4: 2,1$ nadph, and 2 nadh) required to produce acetate 1 ATP and 6 reducing equivalents ( $1 \mathrm{fdxr}-4: 2,1$ nadph, and 4 nadh) required to produce ethanol 
Simulations of the acetate kinase knock out under these conditions showed that the essentiality of ack $A$ gene was dependent on the source of electrons ( $\mathrm{CO}$ or $\mathrm{H}_{2}$ ) and the cofactor specificity of the bifurcating hydrogenase. When $\mathrm{CO}$ is the sole electron donor, all 6 required reducing equivalents are obtained solely in the form of low-potential reduced ferredoxin. Hence, flux is driven through the Rnf complex to get the necessary NADH for the Wood-Ljungdahl pathway and ethanol formation. Since the Rnf complex couples proton translocation with the oxidation of ferredoxin, this results in the formation of the required ATP to fix $\mathrm{CO}_{2}$ using the Wood-Ljungdahl pathway (Figure 5B).

When $\mathrm{H}_{2}$ is the electron donor, and the bifurcating hydrogenase is NADP dependent, the 6 reducing equivalents are obtained in an equimolar split of NADPH and ferredoxin (Figure 5C). The Nfn complex ensures conversion of $2 \mathrm{NADPH}$ to $1 \mathrm{NADH}$ and 1 reduced ferredoxin. However, to get the required balance of ferredoxin and $\mathrm{NADH}$, there is a requirement of flux through the proton translocating Rnf complex that interconverts ferredoxin and NADH. While converting 3 ferredoxin to $3 \mathrm{NADH}$, the proton motive force at the Rnf complex enables the generation of 1.5 ATP. This ensures that carbon fixation and ethanol production is feasible in the absence of $a c k A$. However, if the bifurcating hydrogenase is $\mathrm{NADH}$ dependent, the required 6 reducing equivalents from this reaction are obtained in the form of $3 \mathrm{NADH}$ and 3 ferredoxin (Figure 5D). The Nfn complex and Rnf complex ensure the interconversion of these reducing equivalents to obtain the necessary NADPH and NADH. However, due to the lower demand for NADH in this scenario, the flux through the proton-translocating Rnf complex is reduced, resulting in the generation of only 0.75 ATP.

This ATP generation due to the lower flux through Rnf complex explains the infeasibility of autotrophic growth of the ackA mutant on $\mathrm{H}_{2} / \mathrm{CO}_{2}$ if the cofactor specificity of the bifurcating hydrogenase is NADH. Similarly, since $\mathrm{H}_{2}$ is a major component of syngas along with $\mathrm{CO}$, this impact of the cofactor specificity of the bifurcating hydrogenase on energy conservation during autotrophic growth is fundamental to engineering acetogens for the production of desired compounds from syngas.

\section{Conclusions}

This study presents the first genome-scale metabolic network for an acetogen. The model is predictive of the metabolic capabilities of C. ljungdahlii and together with the recently developed genetic system [35] can aid in the characterization of its metabolic phenotype and guide engineering strategies. For instance, C. ljungdahlii is an important organism in the conversion of synthesis gas. Eliminating acetate production is typically the first choice when engineering acetogens like C. ljungdahlii for the production of chemicals like ethanol. Our results highlight the genetic and energetic constraints while rerouting production of target molecules away from acetate. Therefore, engineering strategies have to account for the appropriate cofactor dependencies and the specific energy conservation mechanisms employed depending on the electron source. Furthermore, C. ljungdahlii is also capable of microbial electrosynthesis, thereby fixing $\mathrm{CO}_{2}$ using electrons directly provided by an electrode [2]. This metabolic model can be extended to obtain insights into potential electron transfer components involved in microbial electrosynthesis. Modeling microbial electrosynthesis would require a systematic evaluation of the different electron transfer pathways through potential carriers such as cytochromes, quinones, or flavins. So far none of these redox active molecules have been identified in C. ljungdahlii. Preliminary studies using a quinone extraction procedure and LCMS have hinted at the presence of a menaquinone-like derivative in C. ljungdahlii. While beyond the scope of this study, a thorough investigation of the structure of this molecule and its potential role in electron transfer could significantly aid modeling and characterization of microbial electrosynthesis. The model could also serve as the basis for gaining further insight into the metabolism of other acetogens. Given the different energy conservation mechanisms adopted by acetogens like M. thermoacetica, the model enables a systematic comparison of the functional impact of these different mechanisms on acetogenic metabolism in general. Overall, these representative examples emphasize how the detailed metabolic reconstruction of an acetogen can aid in the discovery of new function and guiding strain-design strategies for advanced bioproduction from waste streams. 
A Heterotrophic growth on fructose : Feasible

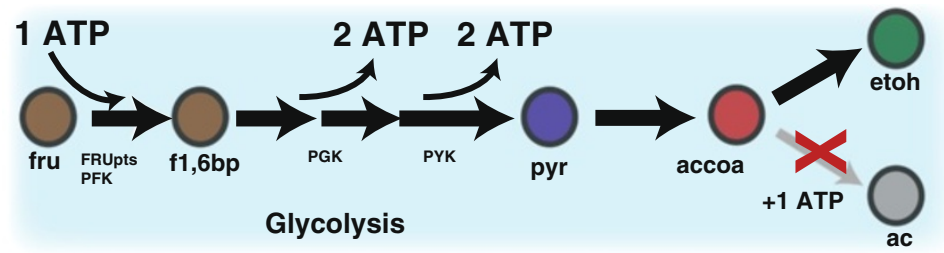

B Autotrophic growth with $\mathrm{CO}$ as electron donor : Feasible

CODH

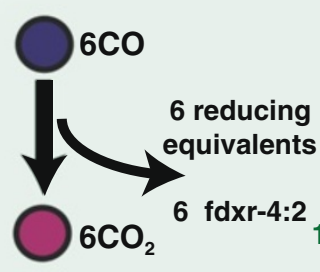

1 fdxr-4:2 + 4 NADH+ $1 \mathrm{NADPH}+2.25$ ATP
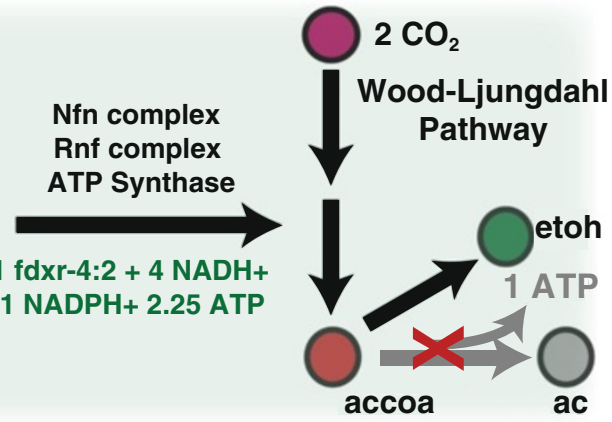

C Autotrophic growth with $\mathrm{H}_{2}$ as electron donor NADPH-specific bifurcating hydrogenase: Feasible
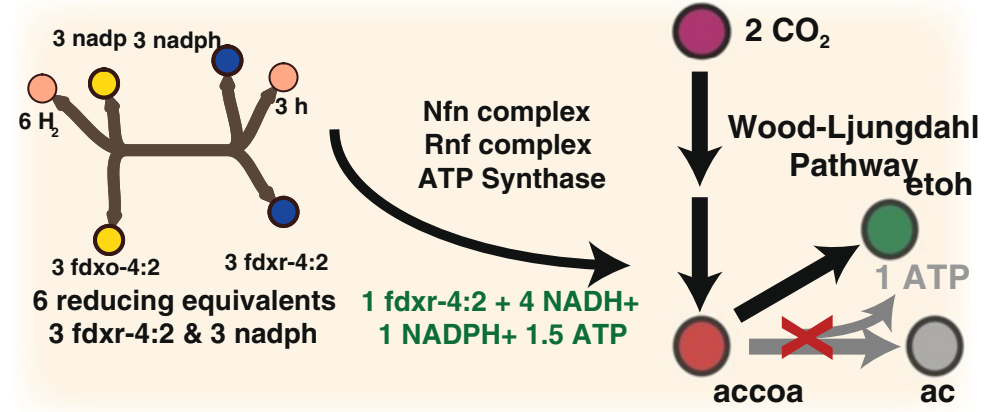

D Autotrophic growth with $\mathrm{H}_{2}$ as electron donor NADH-specific bifurcating hydrogenase: Infeasible
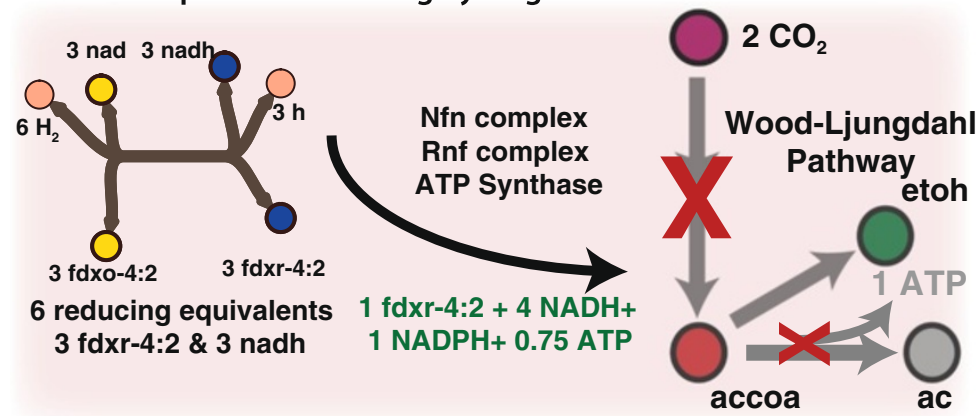

Figure 5 Insights from modeling genetic perturbation into aspects of energy conservation in C. ljungdahlii. (A) Deletion of acetate kinase during growth on fructose shifts the flux from acetate towards ethanol. (B) Growth is feasible when $\mathrm{CO}$ is the electron donor despite the deletion of acetate kinase. (C) Growth is feasible when $\mathrm{H}_{2}$ is the electron donor despite the deletion of acetate kinase if the bifurcating hydrogenase is NADPH dependent. (D) Acetate kinase is essential for autotrophic growth with $\mathrm{H}_{2}$ as the electron donor if the bifurcating hydrogenase is NADH dependent. Modeling analysis reveals an insufficient ATP in the absence of ackA if the hydrogenase is NADH dependent (0.75 ATP). However, if the bifurcating hydrogenase is NADP dependent, sufficient ATP (1.5 ATP) is generated to enable ethanol production in the absence of ackA. 


\section{Methods}

\section{Reconstruction process}

The genome-scale metabolic network for C. ljungdahlii was reconstructed using a four-step integrative reconciliatory workflow involving four published models of related Clostridia species and two draft models. The first draft metabolic model was generated using the ModelSEED database [13]. This draft model is generated automatically in a high-throughput manner by comparing the existing genome annotations in the SEED database. To complement this, we generated another automated draft model that relies on a database of curated genome-scale models. This was done using the AutoModel functionality of SimPheny (Genomatica, San Diego). In addition to these two draft models, homologs to C. ljungdahlii genes were identified in published genome-scale reconstructions of related Clostridia species (C. acetobutylicum [14,15], $C$. thermocellum [16], and C. beijerenckii [17]) using the Smith-Waterman alignment. A $60 \%$ amino acid sequence identity was employed to identify C. ljungdahlii homologs in the other Clostridia genomes. The final curated reconstruction was built in the SimPheny platform (Genomatica, San Diego) to ensure that the reactions in the network were all elementally and charge balanced.

\section{Generation of the biomass objective function}

The Biomass Objective Functions for C. ljungdahlii was formulated using a previous template [36]. The protein content was determined to be $43 \%$ and for the rest of the macromolecular breakdown, the biomass content previously determined for other Gram-positive bacteria, B. subtilis and C. beijerenckii, was used [17,37]. However, for modeling teichoic acid composition, the distribution based on $S$. aureus was used due to similarity in terms of low $\mathrm{G}+\mathrm{C}$ content [38]. It should be noted that prediction of growth rate and unmeasured uptake rates are relatively insensitive to realistic variations in biomass macromolecular weight fractions [39].

\section{Flux balance analysis simulations}

The reconstructed metabolic network was represented in a mathematical format in a stoichiometric matrix $\mathrm{S}$, where the rows correspond to the metabolites and columns correspond to the reactions in the network. Flux Balance Analysis simulations were carried out as described previously [40] using the COBRA Toolbox [41] and the SimPheny framework (Genomatica, Inc., San Diego, CA) was used for simulations. The objective used in the simulations was maximizing growth through the biomass objective function. In silico simulations of gene knockout was carried out using the "singleGeneDeletion" function of the COBRA toolbox [41]. The metabolic models for simulating heterotrophic and autotrophic growth are available for download (Additional file 5: Model 1 and Additional file 6: Model 2).

\section{Bacterial growth conditions and chemical analysis}

Clostridium ljungdahlii (ATCC 55383) was grown in $125 \mathrm{~mL}$ serum bottles under anaerobic conditions containing $100 \mathrm{~mL}$ of PETC medium (ATCC medium 1754) at $37^{\circ} \mathrm{C}$. For growth experiments with nitrate, $1 \mathrm{~mL}$ of an anoxic $\mathrm{NaNO}_{3}$ stock solution $(1 \mathrm{M})$ was added to the medium. Ammonium was omitted in some of the experiments from the medium containing nitrate. Growth was routinely determined by measurement of the $\mathrm{OD}_{600}$. Hydrogen and other gases in the cultures were quantified using gas chromatography as previously described [42]. Concentrations of fructose, acetate, and ethanol were determined by high-performance liquid chromatography (Waters) as previously described [43]. Detection was performed by UV absorption at $410 \mathrm{~nm}$. Nitrate, nitrite, and ammonia concentrations were analyzed using an automated segmented flow analyzer (AA3 HR, SEAL Analytical). Ammonium concentrations were determined by flow injection analysis modified for small sample volumes [44]. The sum of nitrate and nitrite was determined spectrophotometrically after reduction of samples with cadmium [45]. The procedure was identical for determination of nitrite but without the cadmium reduction step.

\section{Transcriptomic profiling of $C$. ljungdahlii}

The transcriptome of C. ljungdahlii was profiled under three different growth conditions: heterotrophically on fructose, heterotrophically on fructose with nitrate as the sole nitrogen source, and autotrophically on $\mathrm{H}_{2} / \mathrm{CO}_{2}$. For each of these conditions, total RNA was isolated from $C$. ljungdahlii cells growing in mid-log phase $\left(\mathrm{OD}_{600}\right.$ of $\sim 0.4$ for both the heterotrophic conditions and an $\mathrm{OD}_{600}$ of $\sim 0.09$ for the autotrophic condition on $\mathrm{H}_{2} / \mathrm{CO}_{2}$ ) using the QIAGEN RNeasy Mini kit with on-column DNase I (QIAGEN) treatment. rRNA was subtracted from $2.5 \mu \mathrm{g}$ of total RNA using the Gram-Positive Ribo-Zero rRNA removal kit (Epicentre). Then, paired-end, strand specific RNA sequencing (RNA-seq) was performed using the dUTP method $[46,47]$ with the following changes. The rRNA subtracted RNA was fragmented with RNA fragmentation reagents (Ambion) for 3 minutes at $70^{\circ} \mathrm{C}$. To synthesize first strand cDNA, random hexamer primers were used (Invitrogen). Final libraries were assayed for quality using High Sensitivity DNA chips on a Bioanalyzer (Agilent) and quantified using a Qubit (Invitrogen). A total of $12.5 \mathrm{pM}$ was loaded onto a MiSeq (Illumina) to yield 31 bp paired-end reads.

The RNA-seq reads were aligned to the genome sequence of C. ljungdahlii (RefSeq: NC_014328.) using Bowtie [48] with two mismatches allowed per read alignment. 
To estimate transcript abundances, FPKM values were calculated for the protein-coding genes using Cufflinks [49] with appropriate parameters set for the strandspecific library type and upper-quartile normalization. The annotation from NCBI was used for transcript quantification. Differential expression analysis was carried out using cuffdiff, with upper-quartile normalization and appropriate parameters set for strand-specific library type. A fold change of greater than 2-fold and false discovery rate cutoff of 0.05 was used to determine significant differential expression.

\section{Additional files}

Additional file 1: Figure S1. Physiological growth screen of $C$. ljungdahlii grown on fructose. Shown are $\mathrm{OD}_{600}$, fructose, and acetate measurements. Error bars represent average of triplicate measurements.

Additional file 2: Table S1. Flux distribution differences when C. ljungdahlii is simulated with and without nitrate grown on fructose.

Additional file 3: Table S2. Differential expression of C. ljungdahlii grown on fructose and autotrophically on $\mathrm{H}_{2} / \mathrm{CO}_{2}$.

Additional file 4: Figure S2. RNA-seq reads from transcriptome profiling of $\mathrm{C}$. ljungdahlii during autotrophic growth on $\mathrm{H}_{2} / \mathrm{CO}_{2}$ showing cotranscription of MetF and MetV.

Additional file 5: Model 1. Metabolic network of C. ljungdahlii. The model file is preset with constraints to simulate heterotrophic growth on fructose. This file can be used with COBRA toolbox in MATLAB.

Additional file 6: Model 2. Metabolic network of C. ljungdahlii. The model file is preset with constraints to simulate autotrophic growth on $\mathrm{H}_{2} / \mathrm{CO}_{2}$. This file can be used with COBRA toolbox in MATLAB.

\section{Competing interests}

The authors declare that they have no competing interest.

\section{Authors' contributions}

$H N$, DRL, and KZ conceived and designed the study. HN, JN, and AE performed the reconstruction. MS and $\mathrm{HL}$ performed the experiments. $\mathrm{HN}$ carried out the simulations and analysis. HN and KZ wrote the manuscript. All authors have read and approved the manuscript.

\section{Acknowledgements}

We thank Malllory Embree (UCSD, Bioengineering) for technical help related to transcriptomics and Adam M. Feist (UCSD, Bioengineering) for assisting with reconstruction of the metabolic model. We are grateful to Susan Becker (UCSD, Scripps Institution of Oceanography) for the analysis of nitrogen compounds in the samples. The work was supported by the Advanced Research Projects Agency-Energy (ARPA-E), U.S. Department of Energy, under Award Numbers DE-AR0000087 and DE-AR0000159 and by the Office of Science (BER), U.S. Department of Energy, Award Number DE-SC0004917. H. Latif was supported through the National Science Foundation Graduate Research Fellowship under grant DGE1144086.

\section{Author details}

${ }^{1}$ Department of Bioengineering, University of California San Diego, La Jolla, CA, USA. ${ }^{2}$ Department of Microbiology, University of Massachusetts, Amherst, MA, USA.

Received: 23 August 2013 Accepted: 21 November 2013

Published: 25 November 2013

\section{References}

1. Drake HL, Gössner AS, Daniel SL: Old acetogens, new light. Ann N Y Acad Sci 2008, 1125:100-128.
2. Nevin KP, Hensley SA, Franks AE, Summers ZM, Ou J, Woodard TL, Snoeyenbos-West OL, Lovley DR: Electrosynthesis of organic compounds from carbon dioxide ls catalyzed by a diversity of acetogenic microorganisms. Appl Environ Microbiol 2011, 77:2882-2886.

3. Nevin KP, Woodard TL, Franks AE, Summers ZM, Lovley DR: Microbial electrosynthesis: feeding microbes electricity to convert carbon dioxide and water to multicarbon extracellular organic compounds. MBio 2010, 1:e00103-e00110.

4. Curtis T, Daran J-M, Pronk JT, Frey J, Jansson JK, Robbins-Pianka A, Knight R, Schnürer A, Smets BF, Smid EJ, et al: Crystal ball- 2013. J Microbial Biotechnol 2013, 6:3-16.

5. Martin WF: Hydrogen, metals, bifurcating electrons, and proton gradients: the early evolution of biological energy conservation. FEBS Lett 2012, 586:485-493.

6. Gencic S, Duin EC, Grahame DA: Tight coupling of partial reactions in the acetyl-CoA decarbonylase/synthase (ACDS) multienzyme complex from Methanosarcina thermophila: acetyl C-C bond fragmentation at the a cluster promoted by protein conformational changes. J Biol Chem 2010, 285:15450-15463.

7. Poehlein A, Schmidt $S$, Kaster A-K, Goenrich $M$, Vollmers J, Thürmer A, Bertsch J, Schuchmann K, Voigt B, Hecker M, et al: An ancient pathway combining carbon dioxide fixation with the generation and utilization of a sodium ion gradient for ATP synthesis. PLOS One 2012, 7:e33439

8. Buckel W, Thauer RK: Energy conservation via electron bifurcating ferredoxin reduction and proton/ $\mathrm{Na}(+)$ translocating ferredoxin oxidation. Biochim Biophys Acta 2012, 8:94-113.

9. Lewis $N E$, Nagarajan $H$, Palsson $B \varnothing$ : Constraining the metabolic genotype-phenotype relationship using a phylogeny of in silico methods. Nat Rev Microbiol 2012, 10:291-305.

10. McCloskey D, Palsson B $\varnothing$, Feist AM: Basic and applied uses of genome-scale metabolic network reconstructions of Escherichia coli. Mol Syst Biol 2013, 9:661

11. Yim H, Haselbeck R, Niu W, Pujol-Baxley C, Burgard A, Boldt J, Khandurina J, Trawick JD, Osterhout RE, Stephen R, et al: Metabolic engineering of Escherichia coli for direct production of 1,4-butanediol. Nat Chem Biol 2011, 7:445-452

12. Köpke M, Held C, Hujer S, Liesegang H, Wiezer A, Wollherr A, Ehrenreich A, Liebl W, Gottschalk G, Dürre P: Clostridium ljungdahlii represents a microbial production platform based on syngas. Proc Natl Acad Sci USA 2010, 107:13087-13092.

13. Henry CS, DeJongh M, Best AA, Frybarger PM, Linsay B, Stevens RL: High-throughput generation, optimization and analysis of genome-scale metabolic models. Nat Biotechnol 2010, 28:977-982.

14. Lee J, Yun H, Feist AM, Palsson BØ, Lee SY: Genome-scale reconstruction and in silico analysis of the Clostridium acetobutylicum ATCC 824 metabolic network. Appl Microbiol Biotechnol 2008, 80:849-862.

15. Senger RS, Papoutsakis ET: Genome-scale model for Clostridium acetobutylicum: part I. Metabolic network resolution and analysis. Biotechnol Bioeng 2008, 101:1036-1052.

16. Roberts SB, Gowen CM, Brooks JP, Fong SS: Genome-scale metabolic analysis of Clostridium thermocellum for bioethanol production. BMC Syst Biol 2010, 4:31.

17. Milne CB, Eddy JA, Raju R, Ardekani S, Kim P-J, Senger RS, Jin Y-S, Blaschek HP Price ND: Metabolic network reconstruction and genome-scale model of butanol-producing strain Clostridium beijerinckii NCIMB 8052. BMC Syst Biol 2011, 5:130.

18. Kanehisa M, Goto S: KEGG: kyoto encyclopedia of genes and genomes. Nucleic Acids Res 2000, 28:27-30.

19. Orth JD, Palsson $B \varnothing$ : Systematizing the generation of missing metabolic knowledge. Biotechnol Bioeng 2010, 107:403-412.

20. Reed JL, Patel TR, Chen KH, Joyce AR, Applebee MK, Herring CD, Bui OT, Knight EM, Fong SS, Palsson $B \varnothing$ : Systems approach to refining genome annotation. Proc Natl Acad Sci USA 2006, 103:17480-17484.

21. Tanner RS, Miller LM, Yang D: Clostridium ljungdahlii sp. nov., an acetogenic species in clostridial rRNA homology group I. Int J Syst Bacteriol 1993, 43:232-236.

22. Köpke M, Mihalcea C, Liew F, Tizard JH, Ali MS, Conolly JJ, Al-Sinawi B, Simpson SD: 2,3-Butanediol production by acetogenic bacteria, an alternative route to chemical synthesis, using industrial waste gas. Appl Environ Microbiol 2011, 77:5467-5475. 
23. Campbell BJ, Smith $J$, Hanson TE, Klotz MG, Stein LY, Lee CK, Wu D, Robinson JM, Khouri HM, Eisen JA, Cary SC: Adaptations to submarine hydrothermal environments exemplified by the genome of Nautilia profundicola. PLOS Genet 2009, 5:e1000362.

24. Hasan SM, Hall JB: The physiological function of nitrate reduction in Clostridium perfringens. J Gen Microbiol 1975, 87:120-128.

25. Ragsdale SW, Pierce E: Acetogenesis and the Wood-Ljungdahl pathway of $\mathrm{CO}_{2}$ fixation. Biochim Biophys Acta Proteins Proteomics 2008, 1784:1873-1898.

26. Bar-Even A: Does acetogenesis really require especially low reduction potential? Biochim Biophys Acta 2012. 10.1016/j.bbabio.2012.1010.1007.

27. Ljungdahl LG: The autotrophic pathway of acetate synthesis in acetogenic bacteria. Annu Rev Microbiol 1986, 40:415-450.

28. Tremblay PL, Zhang T, Dar SA, Leang C, Lovley DR: The Rnf complex of Clostridium ljungdahlii is a proton-translocating ferredoxin: NAD + oxidoreductase essential for autotrophic growth. MBio 2012, 4:e00406-e00412

29. Wang S, Huang H, Moll J, Thauer RK: NADP + reduction with reduced ferredoxin and NADP + reduction with NADH are coupled via an electron-bifurcating enzyme complex in Clostridium kluyveri. J Bacteriol 2010, 192:5115-5123.

30. Huang $\mathrm{H}$, Wang $\mathrm{S}$, Moll J, Thauer RK: Electron bifurcation involved in the energy metabolism of the acetogenic bacterium Moorella thermoacetica growing on glucose or $\mathrm{H}_{2}$ plus $\mathrm{CO}_{2}$. J Bacteriol 2012, 194:3689-3699.

31. Wang S, Huang H, Kahnt J, Mueller AP, Köpke M, Thauer RK: NADP-specific electron-bifurcating [FeFe]-hydrogenase in a functional complex with formate dehydrogenase in Clostridium autoethanogenum grown on $\mathrm{CO}$. J Bacteriol 2013, 195:4373-4386.

32. Wu M, Ren Q, Durkin AS, Daugherty SC, Brinkac LM, Dodson RJ, Madupu R, Sullivan SA, Kolonay JF, Haft DH, et al: Life in hot carbon monoxide: the complete genome sequence of Carboxydothermus hydrogenoformans Z-2901. PLOS Genet 2005, 1:e65

33. Tan Y, Liu J, Chen $X$, Zheng H, Li F: RNA-seq-based comparative transcriptome analysis of the syngas-utilizing bacterium Clostridium ljungdahlii DSM 13528 grown autotrophically and heterotrophically. Mol Bio Syst 2013, 9:2775-2784

34. Schuchmann $K$, Müller $V$ : A bacterial electron-bifurcating hydrogenase. J Biol Chem 2012, 287:31165-31171.

35. Leang C, Ueki T, Nevin KP, Lovley DR: A genetic system for Clostridium ljungdahlii: a chassis for autotrophic production of biocommodities and a model homoacetogen. Appl Environ Microbiol 2013, 79:1102-1109.

36. Feist $\mathrm{AM}$, Palsson $\mathrm{B} \varnothing$ : The biomass objective function. Curr Opin Microbiol 2010, 13:344-349.

37. Oh Y-K, Palsson BØ, Park SM, Schilling CH, Mahadevan R: Genome-scale reconstruction of metabolic network in Bacillus subtilis based on high-throughput phenotyping and gene essentiality data. $J$ Biol Chem 2007, 282:28791-28799.

38. Xia G, Kohler T, Peschel A: The wall teichoic acid and lipoteichoic acid polymers of Staphylococcus aureus. Int J Med Microbiol 2010, 300:148-154.

39. Feist AM, Henry CS, Reed JL, Krummenacker M, Joyce AR, Karp PD, Broadbelt $L$, Hatzimanikatis $\mathrm{V}$, Palsson $\mathrm{B} \varnothing$ : A genome-scale metabolic reconstruction for Escherichia coli K-12 MG1655 that accounts for 1260 ORFs and thermodynamic information. Mol Syst Biol 2007, 3:121.

40. Orth JD, Thiele I, Palsson $B \varnothing$ : What is flux balance analysis? Nat Biotechnol 2010, 28:245-248

41. Schellenberger J, Que R, Fleming RMT, Thiele I, Orth JD, Feist AM, Zielinski DC, Bordbar A, Lewis NE, Rahmanian S, et al: Quantitative prediction of cellular metabolism with constraint-based models: the COBRA toolbox v2.0. Nat Protoc 2011, 6:1290-1307.

42. Gong Y, Ebrahim A, Feist AM, Embree M, Zhang T, Lovley D, Zengler K. Sulfide-driven microbial electrosynthesis. Environ Sci Tech 2013, 47:568-573.

43. Portnoy VA, Herrgard MJ, Palsson B $\varnothing$ : Aerobic fermentation of D-glucose by an evolved cytochrome oxidase-deficient Escherichia coli strain. Appl Environ Microbiol 2008, 74:7561-7569.

44. Aminot A, Kerouel R, Birot D: A flow injection-fluorometric method for the determination of ammonium in fresh and saline waters with a view to in situ analyses. Water Res 2001, 35:1777-1785.

45. Jones MN: Nitrate reduction by shaking with cadmium: alternative to cadmium columns. Water Res 1984, 18:643-646.
46. Parkhomchuk D, Borodina T, Amstislavskiy V, Banaru M, Hallen L, Krobitsch S, Lehrach $\mathrm{H}$, Soldatov A: Transcriptome analysis by strand-specific sequencing of complementary DNA. Nucleic Acids Res 2009, 37:e123.

47. Zhong S, Joung J-G, Zheng Y, Chen Y-R, Liu B, Shao Y, Xiang JZ, Fei Z Giovannoni JJ: High-throughput Illumina strand-specific RNA sequencing library preparation. Cold Spring Harb Protoc 2011, 2011:940-949.

48. Langmead B, Trapnell C, Pop M, Salzberg SL: Ultrafast and memory-efficient alignment of short DNA sequences to the human genome. Genome Biol 2009, 10:R25

49. Trapnell C, Williams BA, Pertea G, Mortazavi A, Kwan G, van Baren MJ, Salzberg SL, Wold BJ, Pachter L: Transcript assembly and quantification by RNA-Seq reveals unannotated transcripts and isoform switching during cell differentiation. Nat Biotechnol 2010, 28:511-515.

doi:10.1186/1475-2859-12-118

Cite this article as: Nagarajan et al:: Characterizing acetogenic metabolism using a genome-scale metabolic reconstruction of Clostridium ljungdahlii. Microbial Cell Factories 2013 12:118.

\section{Submit your next manuscript to BioMed Central and take full advantage of:}

- Convenient online submission

- Thorough peer review

- No space constraints or color figure charges

- Immediate publication on acceptance

- Inclusion in PubMed, CAS, Scopus and Google Scholar

- Research which is freely available for redistribution

Submit your manuscript at www.biomedcentral.com/submit
C Biomed Central 